\title{
SRMBUILDER: A USER-FRIENDLY TOOL FOR SELECTED REACTION MONITORING DATA ANALYSIS
}

\author{
QUANHU SHENG*, ${ }^{*}$, CHAOCHAO $\mathrm{WU}^{\dagger}, \boldsymbol{\uparrow}$, \\ ZHIDUAN SU ${ }^{\ddagger}$ and RONG ZENG $§$ \\ Key Laboratory of Systems Biology \\ Institute of Biochemistry and Cell Biology \\ Shanghai Institutes for Biological Sciences \\ Chinese Academy of Sciences, Shanghai, China \\ *qhsheng@sibs.ac.cn \\ ${ }^{\dagger}$ ccwu@sibs.ac.cn \\ ${ }^{\ddagger}$ suzhiduan@sibs.ac.cn \\ §zr@sibs.ac.cn
}

Received 25 July 2011

Revised 5 September 2011

Accepted 15 September 2011

\begin{abstract}
With high sensitivity and reproducibility, selected reaction monitoring (SRM) has become increasingly popular in proteome research for targeted quantification of low abundance proteins and post translational modification. SRM is also well accepted in other mass-spectrometry based research areas such as lipidomics and metabolomics, which necessitates the development of easy-to-use software for both post-acquisition SRM data analysis and quantification result validation. Here, we introduce a software tool SRMBuilder, which can automatically parse SRM data in multiple file formats, assign transitions to compounds, match light/heavy transition/compound pairs and provide a user-friendly graphic interface to manually validate the quantification result at transition/compound/sample level. SRMBuilder will greatly facilitate processing of the post-acquisition data files and validation of quantification result for SRM. The software can be downloaded for free from http://www.proteomics.ac.cn/ software/proteomicstools/index.htm as part of the software suite Proteomics Tools.
\end{abstract}

Keywords: SRM; SRMBuilder; quantification; mass spectrometry; targeted proteomics.

\section{Introduction}

Shotgun proteomics approach has been widely used for the large scale identification and quantification of peptides and proteins, which demonstrates a combination of comprehensiveness and accuracy. ${ }^{1}$ However, in spite of its significant advantages, shotgun proteomics becomes dim when considering reproducibility and sensitivity in comparison with targeted proteomics. ${ }^{2}$ Selected reaction monitoring (SRM)

\footnotetext{
『Both authors contributed equally to this work.

$\S$ Corresponding author.
} 
represents a powerful contrivance of targeted proteomics. ${ }^{3}$ Recently, SRM has been shown with great potential for detecting and quantifying low-abundance protein biomarkers in plasma, ${ }^{4,5}$ for elucidating metabolic pathways ${ }^{6}$ and even for profiling proteome at full dynamic range. ${ }^{7}$ The application of SRM has also been widely applied in the field of metabolomics ${ }^{8}$ as well as lipidomics, ${ }^{9}$ especially for the absolute quantification of targeted compounds by the spike-in of a standard compound mixture. ${ }^{10,11}$

In a general SRM-based quantitative proteomics experiment, multiple peptides with high mass spectrometry detectability will be selected for each protein and multiple transitions for each peptide will be designed from in silicon prediction ${ }^{12}$ or spectrum-library based identification result. ${ }^{3}$ Thus for a large scale experiment containing thousands of transitions, each sample may be divided into several runs and each run contains hundreds of transitions due to the limitation of the instrument capacity. ${ }^{6}$

With the rapid development of SRM-based targeted proteomics, many tools have been published such as TIQAM, ${ }^{13}$ MRMer, ${ }^{14}$ MaRiMba, ${ }^{15}$ Skyline, ${ }^{16}$ and ATAQS. ${ }^{17,18}$ TIQAM is a desktop software suite including TIQAM-digestor for transition generation and TIQAM-viewer for data validation, however, only the data acquired from Q-TRAP 4000 is supported. MRMer is a java-based application for SRM data validation, but only analyzes one file at each time and thus not suitable for large scale SRM experiment. MaRiMba is designed only for transition generation of proteomics but not post-acquisition SRM data analysis. Skyline is also a windows client application and supports the entire SRM-based workflow from transition design to data analysis. Since it is completely peptide-based, Skyline is incompatible with the data in lipidomics or metabolomics at present, besides, it cannot analyze the data with decoy transitions that were produced by shifting precursor/product ion masses from normal peptide for the calculation of false discovery rate in SRM experiment. ${ }^{17}$ ATAQS is a web-based platform that supports the team collaboration and data sharing, however, the functionalities for manual validation and the adjustment of elution time windows are missing. Altogether, these software greatly accelerate the exertion of SRM based targeted proteomics, however, it is still highly necessary to develop an easy-to-use software which is compatible with proteomics, lipidomics, metabolomics data and supports multiple data formats, large scale data processing, automatic and manual validation of quantification result.

Here we introduce software named SRMBuilder, which can simultaneously analyze multiple SRM data files, validate light/heavy ratio at transition/compound/sample level and output the quantification result. It supports multiple data formats including mzXml, mzData, Thermo Raw and Agilent.d director. The software can be downloaded for free from http://www.proteomics.ac.cn/ software/proteomicstools/index.htm as part of the software suite Proteomics Tools. 


\section{Implementation}

\subsection{SRMBuilder system overview}

SRMBuilder is developed in the $\mathrm{C \#}$ programming language and compiled in Microsoft Visual Studio 2010 Professional Edition. The developed software is fully compatible with Windows-based operating system with dotNET framework v3.5. The software features an easy installation procedure at Microsoft windows operation system and provides a user-friendly graphic interface.

SRMBuilder includes two modules: SRMDistiller and SRMValidator. SRMDistiller is a highly intelligent module used to extract transition information, gain quantification result and output preliminary results. SRMValidator can further input those preliminary results, and display them in a user-friendly graphic interface that allows users to manually validate the results at the transition/compound/sample level and output the final quantification results.

\subsection{SRMDistiller}

SRMDistiller can automatically extract transition information from raw data files, assign transitions to compounds (usually peptides), match light/heavy compound pairs, calculate light/heavy ratios, combine compound information from the same sample, validate transitions/compounds, and output preliminary results for each sample/replication.

\subsubsection{Transition/compound extraction}

For each data file, three steps are used to extract transition/compound entries.

(1) Each tandem mass spectrum will be separated into multiple transition scans so that each scan includes only one product ion and its corresponding precursor. All transition scans from same data file will be stored in a cache file for the convenience of future analyses.

(2) The transition scans with the same precursor and product ion will be assembled into one transition entry.

(3) Based on a predefined transition file, the co-eluted transition entries with the same precursor will be assigned to one compound entry. For the data from quantitative measurement using labeled internal standards, unlabeled (light) compound entry and the labeled internal standard (heavy) compound entry will be recognized and constructed to a compound pair. The transition entries in the compound pair will be matched and constructed to transition pairs. Each transition pair contains a light transition from light compound and corresponding heavy transition from heavy compound. 


\subsubsection{Peak picking}

Usually, the measurement time window of a transition will be designed larger than the compound's actual elution time window to make sure that all signals from that transition will be monitored. The determination of the true elution time window is highly correlated with the quantification accuracy. Considering that the product ions from the same compound should have the same elution time window, we developed a refining method to determine the correct elution time window for quantification.

(1) For each transition entry, the scan whose product ion intensity is larger than a user-defined threshold will be marked as valid. Here, we provide two options for the user-defined threshold: the multiple folds of the baseline, suitable for the data with a stable baseline; the percentage of the largest ion intensity in the same transition. A peak is constructed from continuous valid scans. If there are multiple peaks, only the one with highest intensity will be kept.

(2) For each product ion pair (transition), the elution time window of transition with the highest ion will be considered as the reference and mapped to the corresponding isotopic pair transition. Then, light/heavy ratio of the product ion pair will be calculated using a linear regression model.

(3) For each compound pair (peptide), the elution time window of the product ion pair with the largest linear regression correlation coefficient will be considered as the reference and mapped to the other product ion pairs. Light/heavy ratio of the compound will be recalculated after the elution time window refinement.

\subsubsection{Sample data construction}

The compound pairs from the files with the same group name, which indicates from the same sample, will be combined together.

\subsubsection{Automatic data filter}

Three fixed criteria can be used to filter out false positive transition pairs: the minimum signal/noise threshold for light and heavy transitions (2, in this work), the minimum linear regression correlation coefficient (0.6, in this work) and the minimum valid scan count (5, in this work). For the data with decoy transitions, transitions will be filtered by the false discovery rate (FDR) 0.05 using the correlation coefficient as the score.

If there are more than three transition pairs of same compound pair passed all fixed criteria and FDR filtering, we assume that at most one transition pair of them is outlier and use following algorithm to distinguish it from others.

(1) Let ratio $\mathrm{R}=\log ($ ratio (light/heavy)) and calculate the mean( $\mathrm{R})$ of all valid transition pairs. 
(2) Let distance $\mathrm{D}=\mid \mathrm{R}$-mean $(\mathrm{R}) \mid$ and find out the transition pair with the largest distance (TPLD).

(3) Excluding the TPLD and assuming the left ratios are from a normal distribution, the probability that the ratio of TPLD is from this normal distribution will be calculated. The TPLD with probability less than $10^{-4}$ will be discarded.

A valid transition pair is defined as the transition pair passed all fixed criteria, FDR filter and outlier deletion. Then, one fixed criteria is used to filter false positive compound pairs: the minimum valid transition pair count (2, in this work).

Finally, each sample's preliminary results will be outputted for additional manual validation.

\subsection{SRMValidator}

SRMValidator can be used to load multiple SRMDistiller output files, manual validate the quantification results at transition/compound/sample levels and output final quantification results.

\subsubsection{Transition level validation}

The automatic procedure may detect wider elution window which includes noise peaks. User can choose correct time range manually based on product ion chromatograph and linear regression result (Fig. 1).

$510.5767-513.9127$
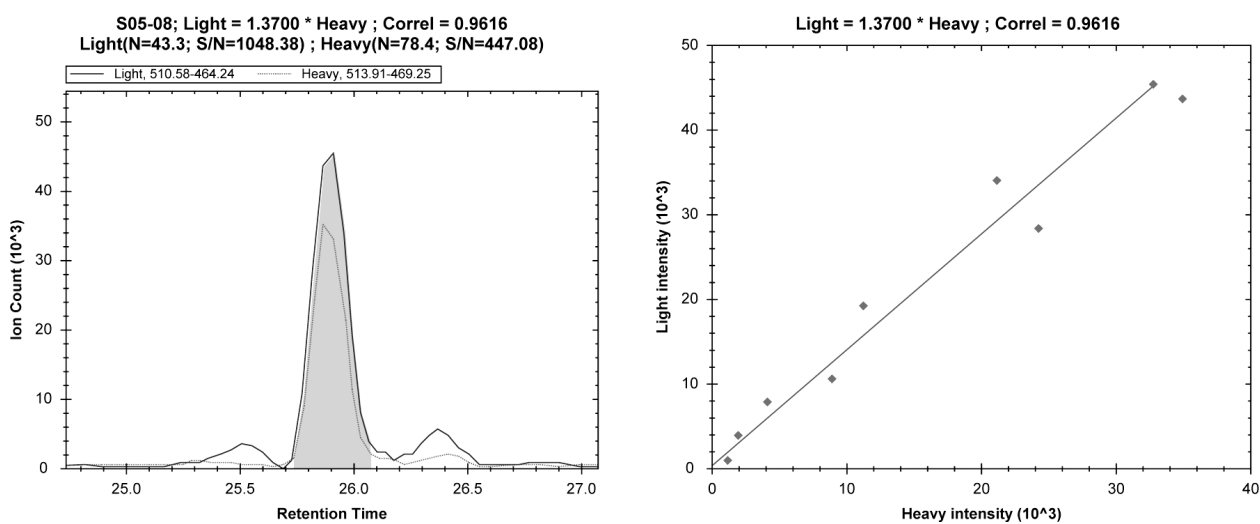

Fig. 1. Transition level validation. The left graph indicates the chromatograph of the transition pair and the right graph show corresponding linear regression results.

\subsubsection{Compound level validation}

Since transition pairs in same compound pair should have similar elution chromatographs, user can determinate the most reliable elution time window of the 

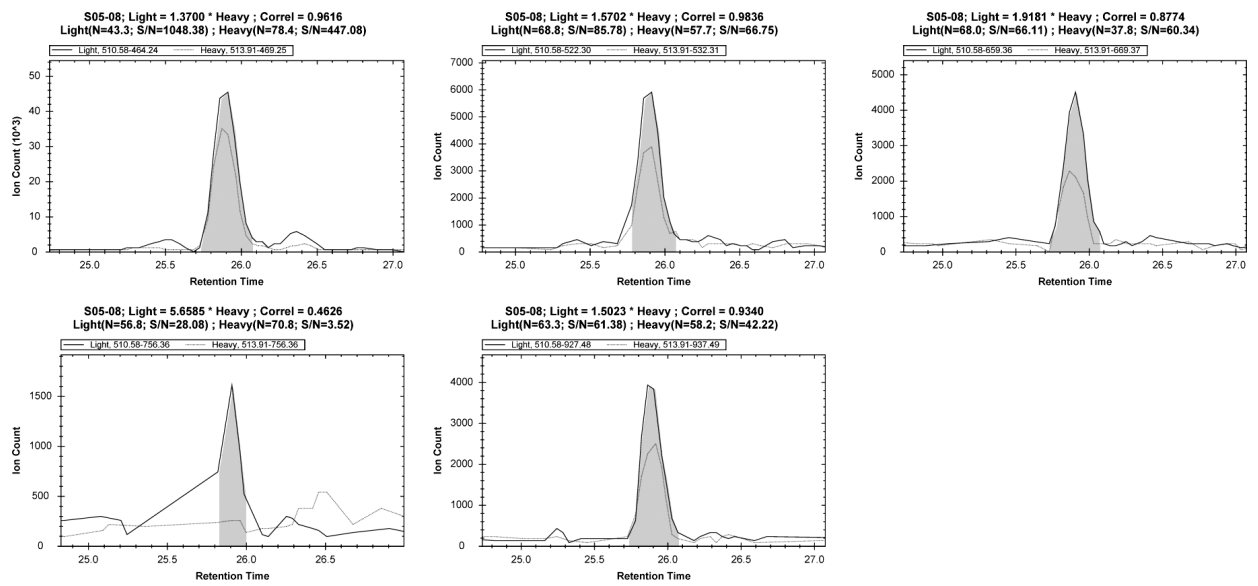

Fig. 2. Compound level validation. Five chromatographs correspond to five transition pairs from same compound.

compound pair based on all transition pair chromatographs (Fig. 2). Once the elution time window of a transition pair is determined, all elution time windows of other transition pairs from the same compound pair will be synchronized simultaneously. Finally, the light/heavy ratios of those transition pairs will be recalculated automatically.

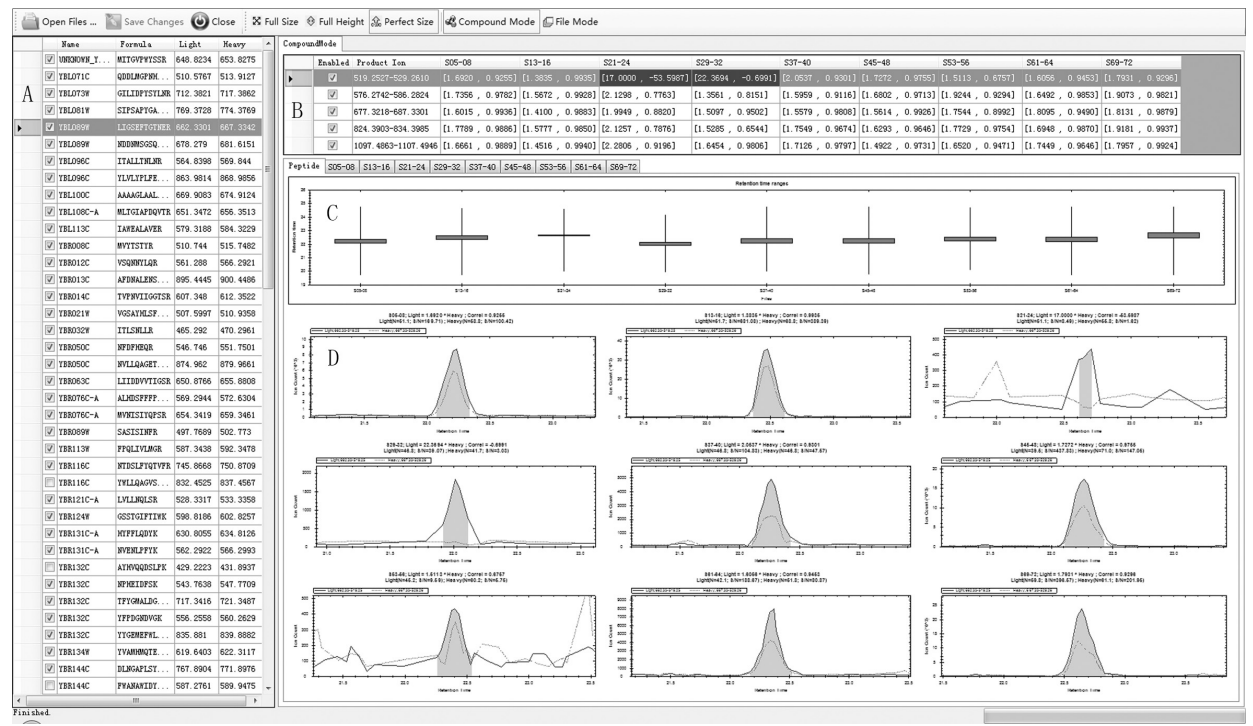

Fig. 3. Sample level validation. (A) Compound list. (B) Transition list with the ratio and the correlation coefficient in each sample. The entry with the correlation coefficient less than a userdefined threshold will be highlighted in red. (C) The elution time windows of current transition in all samples. (D) The chromatographs of the current transition in all samples. 


\subsubsection{Sample level validation}

The quantification results of the transition pair from multiple samples can be compared at same window as illustrates in Fig. 3. The transition pair with lower correlation coefficient will be highlighted. The elution window of each transition pair can be adjusted manually based on cross-sample comparison.

\subsubsection{Export quantification result}

Final quantification result can be exported at two levels: the compound and the transition. Decoy entries and invalid entries can be discarded based on user choice. Besides, chromatograms of selected transitions can be exported as html/image files.

\section{Results and Discussion}

We tested SRMBuilder using the ATAQS yeast dataset ${ }^{18}$ which contained nine samples and each sample contained four data files and 2000 transitions (including 1000 target transitions and 1000 decoy transitions).

\subsection{Refinement result}

Figure 4 illuminates that SRMDistiller detected the actual elution time windows using the refinement algorithm. Even false windows were detected, as Figs. 4A1 and $4 \mathrm{~A} 2$ indicates, it was corrected by the refinement algorithm considering the co-elution features of the transitions from the same compound.

\subsection{Outlier detection}

Figure 5 indicates that SRMDistiller detected the outlier that has high regression correlation coefficients. The exclusion of outliers from the compound ratio calculation can definitely improve the accuracy of quantification.

\subsection{Validation accuracy}

Table 1 shows the statistic result of all nine samples after automatic analysis and filter by SRMDistiller. All results demonstrate high specificity in excluding decoy compounds. There are four results (S05-08, S13-16, S45-48 and S69-72) which achieved high sensitivity (higher than $90 \%$ ) of target compounds detection. Another two (S37-40, S61-64) also achieved good sensitivity (higher than 70\%). The other three (S21-24, S29-32 and S53-56) showed sensitivity lower than 40\%. Manual check of those three results showed low signal to noise level, and the chromatograms of transitions were significantly different to others (Fig. 6). The full version of chromatograms of all samples can be downloaded from our website. The statistic and manual check results indicate that those three samples should be excluded from the analysis. 

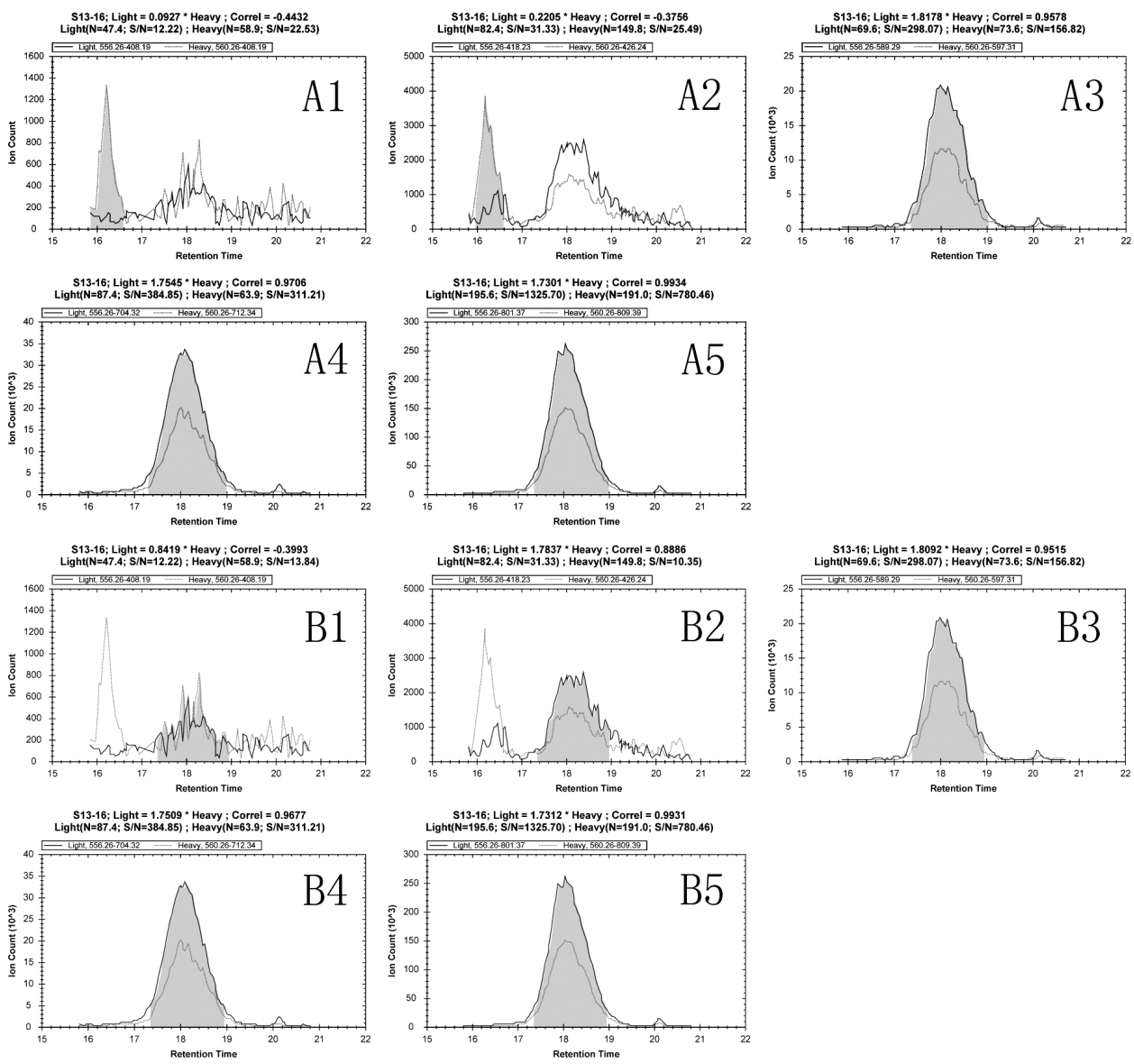

Fig. 4. The SRMBuilder refinement algorithm is capable of selecting the correct elution time window. A1-A5 and B1-B5 indicates five transition pair chromatographs before and after the elution time refinement. A1 and A2 show that false elution time windows were detected. B1 and B2 show that the false windows were corrected by refinement algorithm.

\section{Conclusions}

In this paper, we presented the SRMBuilder software package, which could be used to analyze large scale SRM data from multiple file formats, validate the quantification results either automatically or manually, and export the final quantification results. A user friendly graphic interface was provided for manual check and elution time adjustment at transition/compound/sample level. Since the software is not peptide-based, the whole analysis procedure can be transferred in other SRMbased applications such as lipidomics and metabolomics. The testing result with large scale datasets showed that our software could distinguish decoy compounds from target compounds successfully with high sensitivity and specificity. Therefore, SRMBuilder could facilitate the post-acquisition data analysis procedure of targeted analysis, and a useful replenishment of SRM based software. 

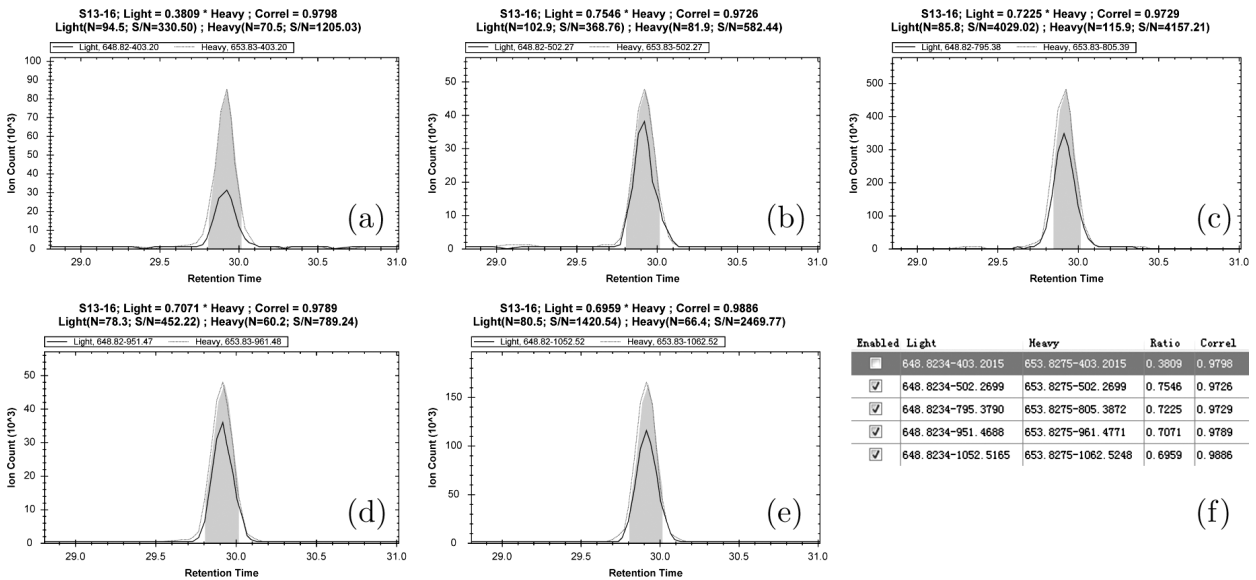

Fig. 5. The SRMBuilder outlier detection algorithm is capable of selecting the outlier with high correlation coefficient. All five transition pairs (a-e) have high correlation coefficients (higher than 0.95). The ratio of highlighted transition pair $(\mathrm{a}, 0.38)$ is significantly different from the other four ratios (b, c, d and e, near 0.70) and was set as invalid (f, the first entry is unchecked).

Table 1. Automatic filter result of all samples.

\begin{tabular}{lccccccccc}
\hline Compound & S05-08 & S13-16 & S21-24 & S29-32 & S37-40 & S45-48 & S53-56 & S61-64 & S69-72 \\
\hline Valid decoy & 1 & 2 & 0 & 0 & 0 & 0 & 0 & 0 & 3 \\
Invalid target & 6 & 5 & 68 & 85 & 27 & 8 & 63 & 16 & 7 \\
Valid target & 94 & 95 & 32 & 15 & 73 & 92 & 37 & 84 & 93 \\
\hline
\end{tabular}
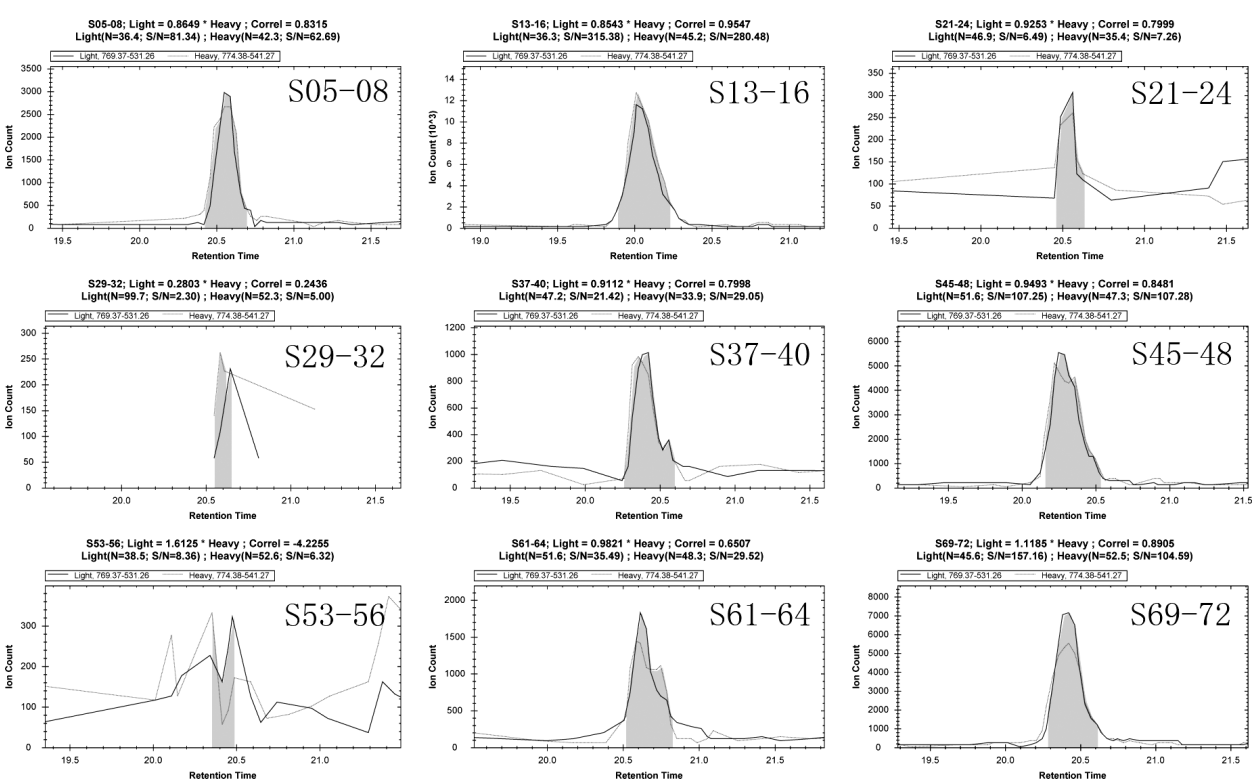

Fig. 6. Chromatograms of compound (YBL081W-SIPSAPYGAYNQNR) in all nine samples. Three chromatographs from S21-24, S29-32 and S53-56 are significantly different with others. 


\section{Acknowledgments}

This work is supported by a grant from Shanghai Institutes for Biological Sciences (2009KIP212); the grants from Ministry of Science and Technology (2010CB912102, 2011CB910200); a grant from the National Natural Science Foundation of China (30821065); and a grant from the Knowledge Innovation Program of the Chinese Academy of Sciences (KSCX1-YW-02). The authors also gratefully acknowledge the support of SA-SIBS scholarship program.

\section{References}

1. Cox J, Mann M, Quantitative, high-resolution proteomics for data-driven systems biology, Annu Rev Biochem 80:273-299, 2011.

2. Duncan MW, Aebersold R, Caprioli RM, The pros and cons of peptide-centric proteomics, Nat Biotechnol 28(7):659-664, 2010.

3. Lange V, Picotti P, Domon B, Aebersold R, Selected reaction monitoring for quantitative proteomics: A tutorial, Mol Syst Biol 4:222, 2008.

4. Keshishian H, Addona T, Burgess M, Mani DR, Shi X, Kuhn E, Sabatine MS, Gerszten RE, Carr SA, Quantification of cardiovascular biomarkers in patient plasma by targeted mass spectrometry and stable isotope dilution, Mol Cell Proteomics 8(10):2339-2349, 2009.

5. Fortin T, Salvador A, Charrier JP, Lenz C, Lacoux X, Morla A, Choquet-Kastylevsky $\mathrm{G}$, Lemoine J, Clinical quantitation of prostate-specific antigen biomarker in the low nanogram/milliliter range by conventional bore liquid chromatography-tandem mass spectrometry (multiple reaction monitoring) coupling and correlation with ELISA tests, Mol Cell Proteomics 8(5):1006-1015, 2009.

6. Costenoble R, Picotti P, Reiter L, Stallmach R, Heinemann M, Sauer U, Aebersold $\mathrm{R}$, Comprehensive quantitative analysis of central carbon and amino-acid metabolism in Saccharomyces cerevisiae under multiple conditions by targeted proteomics, Mol Syst Biol 7:464, 2011.

7. Picotti P, Bodenmiller B, Mueller LN, Domon B, Aebersold R, Full dynamic range proteome analysis of S. cerevisiae by targeted proteomics, Cell 138(4):795-806, 2009.

8. Panusa A, Regazzoni L, Aldini G, Orioli M, Giombini A, Minghetti P, Tranquilli C, Carini M, Urinary profile of methylprednisolone acetate metabolites in patients following intra-articular and intramuscular administration, Anal Bioanal Chem 400(1):255267, 2011.

9. Sato H, Taketomi Y, Isogai Y, Miki Y, Yamamoto K, Masuda S, Hosono T, Arata S, Ishikawa Y, Ishii T, Kobayashi T, Nakanishi H, Ikeda K, Taguchi R, Hara S, Kudo I, Murakami M, Group III secreted phospholipase A2 regulates epididymal sperm maturation and fertility in mice, J Clin Invest 120(5):1400-1414, 2010.

10. Ikeda K, Shimizu T, Taguchi R, Targeted analysis of ganglioside and sulfatide molecular species by LC/ESI-MS/MS with theoretically expanded multiple reaction monitoring, J Lipid Res 49(12):2678-2689, 2008.

11. Deroussent A, Rodriguez S, Martelli S, Seck A, Dubus-Daudigeos E, Desmaele D, Vassal G, Paci A, Quantification of dimethyl-ifosfamide and its N-deschloropropylated metabolites in mouse plasma by liquid chromatography-tandem mass spectrometry, J Chromatogr B Analyt Technol Biomed Life Sci 879(11-12):743-750, 2011.

12. Fusaro VA, Mani DR, Mesirov JP, Carr SA, Prediction of high-responding peptides for targeted protein assays by mass spectrometry, Nat Biotechnol 27(2):190-198, 2009.

13. Lange V, Malmstrom JA, Didion J, King NL, Johansson BP, Schafer J, Rameseder J, Wong CH, Deutsch EW, Brusniak MY, Buhlmann P, Bjorck L, Domon B, 
Aebersold R, Targeted quantitative analysis of Streptococcus pyogenes virulence factors by multiple reaction monitoring, Mol Cell Proteomics 7(8):1489-1500, 2008.

14. Martin DB, Holzman T, May D, Peterson A, Eastham A, Eng J, McIntosh M, MRMer, An interactive open source and cross-platform system for data extraction and visualization of multiple reaction monitoring experiments, Mol Cell Proteomics 7(11):2270-2278, 2008.

15. Sherwood CA, Eastham A, Lee LW, Peterson A, Eng JK, Shteynberg D, Mendoza L, Deutsch EW, Risler J, Tasman N, Aebersold R, Lam H, Martin DB, MaRiMba: A software application for spectral library-based MRM transition list assembly, J Proteome Res 8(10):4396-4405, 2009.

16. MacLean B, Tomazela DM, Shulman N, Chambers M, Finney GL, Frewen B, Kern R, Tabb DL, Liebler DC, MacCoss MJ, Skyline: An open source document editor for creating and analyzing targeted proteomics experiments, Bioinformatics 26(7):966968, 2010.

17. Reiter L, Rinner O, Picotti P, Huttenhain R, Beck M, Brusniak MY, Hengartner MO, Aebersold R, mProphet: Automated data processing and statistical validation for large-scale SRM experiments, Nat Methods, 2011.

18. Brusniak MY, Kwok ST, Christiansen M, Campbell D, Reiter L, Picotti P, Kusebauch U, Ramos H, Deutsch EW, Chen J, Moritz RL, Aebersold R, ATAQS: A computational software tool for high throughput transition optimization and validation for selected reaction monitoring mass spectrometry, BMC Bioinformatics 12(1):78, 2011.

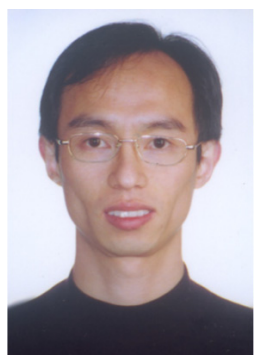

Quanhu Sheng received both his M.Sc. in Biochemistry and Cell Biology and Ph.D. in Bioinformatics from Shanghai Institutes for Biological Sciences (SIBS), Chinese Academy of Sciences (CAS), China, in 2003 and 2009, respectively, and has since been working as associate professor in the Research Center for Proteome Analysis, Key Lab of Systems Biology, SIBS, CAS. He is working primarily for the algorithm and software development for bioinformatics in mass spectrometry based proteomics, glycomics and metabolomics, especially in the field of peptide/protein identification, verification and quantification.

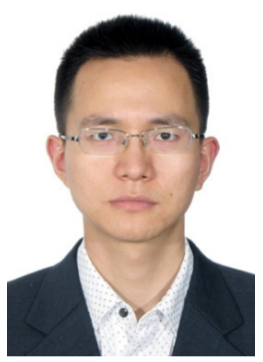

Chaochao Wu obtained his B.Sc. at Zhejiang University, China in 2005. Until now, he has been studying proteomics as a PHD student at the Institute of Biochemistry and Cellular Biology, Shanghai Institutes for Biological Sciences (SIBS), Chinese Academy of Sciences (CAS), China. His current research focused on the application of short-gun proteomics and selected reaction monitoring (SRM) to elucidate the complexity of dynamic metabolic reprogramming during carcinogenesis. He was also interested in detecting and validating new modification sites on low abundant signaling proteins. 

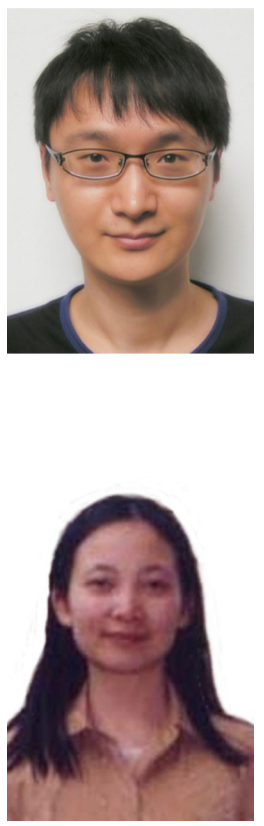

Zhiduan Su graduated with a bachelor's degree in biochemistry from Shandong University (SDU), China, in 2007. Till now, he is a Ph.D student from Key Laboratory of Systems Biology, Institute of Biochemistry and Cell Biology, Shanghai Institutes for Biological Sciences, Chinese Academy of Sciences, China. His research interest is focused on the development and application of methods for identification and quantification of single aminoacid polymorphisms (SAPs) at proteome-level based on shotgun and targeted proteomics strategy.

Rong Zeng is the group leader and professor of Institute of Biochemsitry and Cell Biology. She received her Ph.D. in Biochemistry from Shanghai Institute of Biochemistry, Chinese Academy of Sciences (CAS) in 2000. She leads the Research Center for Proteome Analysis and focuses on the proteomics and protein dynamic behavior. 